\title{
Capillary Structured Suspensions from In Situ Hydrophobized Calcium Carbonate Particles Suspended in a Polar Liquid Media
}

\author{
Timothy S. Dunstan, ${ }^{\dagger} \odot$ Anupam A. K. Das, ${ }^{\dagger} \odot$ Pierre Starck, ${ }^{\ddagger}$ Simeon D. Stoyanov,,${ }^{\S, \|, \perp \odot}$
} and Vesselin N. Paunov*,†

\author{
${ }^{\dagger}$ School of Mathematics and Physical Sciences (Chemistry), University of Hull, Hull HU6 7RX, United Kingdom \\ ${ }^{\ddagger}$ Unilever R\&D Port Sunlight, Quarry Road East, Bebington, CH63 3JW, United Kingdom \\ ${ }^{\S}$ Unilever R\&D Vlaardingen, Olivier van Noortlaan 120, 3133 AT Vlaardingen, The Netherlands \\ "Laboratory of Physical Chemistry and Soft Matter, Wageningen University, 6703 HB Wageningen, The Netherlands \\ ${ }^{\perp}$ Department of Mechanical Engineering, University College London, Torrington Place, London WC1E 7JE, United Kingdom
}

Supporting Information

ABSTRACT: We demonstrate that capillary suspensions can be formed from hydrophilic calcium carbonate particles suspended in a polar continuous media and connected by capillary bridges formed of minute amounts of an immiscible secondary liquid phase. This was achieved in two different polar continuous phases, water and glycerol, and three different oils, oleic acid, isopropyl myristate, and peppermint oil as a secondary liquid phase. The capillary structuring of the suspension was made possible through local in situ hydrophobization of the calcium carbonate particles dispersed in the polar media by adding very small amounts of oleic acid to the secondary liquid phase. We observed a strong increase in the viscosity of the calcium carbonate suspension by several orders of magnitude upon addition of the secondary oil phase compared with the same suspension without secondary liquid phase or without oleic acid. The stability and the rheological properties of the obtained capillary structured materials were studied in relation to the physical properties of the system such as the particle size, interfacial tension between the
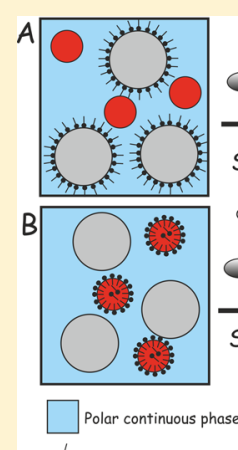

Oleic acid
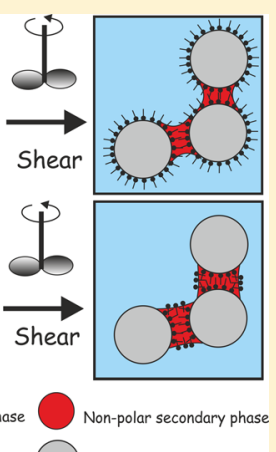
primary and secondary liquid phases, as well as the particle contact angle at this liquidliquid interface. We also determined the minimal concentrations of the secondary liquid phase at fixed particle concentration as well as the minimal particle concentration at fixed secondary phase concentration needed to form a capillary suspension. Capillary suspensions formed by this method can find application in structuring pharmaceutical and food formulations as well as a variety of home and personal care products.

\section{INTRODUCTION}

Capillary structured suspensions are systems with particles dispersed in a continuous liquid phase and stabilized using a minute amount of an immiscible secondary fluid phase. ${ }^{1}$ The addition of the secondary fluid results in the formation of capillary bridges between the particles, which is mainly responsible for the increase in long-term stability of the suspension. ${ }^{2}$ The rheological properties of the capillary suspensions can be altered significantly by extremely small changes in the overall concentration of the secondary immiscible fluid. ${ }^{3-6}$ Capillary structured suspensions have many potential applications from food formulations ${ }^{7}$ to ceramics. ${ }^{8}$ Many different forces influence the rheology and the flow of the suspensions (particles dispersed in a liquid phase), such as van der Waals attraction, electrostatic repulsion, steric interactions, and hydrodynamic and Brownian forces. ${ }^{9-11}$ The addition of a small amount of the secondary immiscible liquid to the suspension results in the conversion of the fluidlike suspension to a gel-like state or from a weak gel-like state to a strong gel-like behavior depending on the particle concentration. ${ }^{7}$ The capillary force plays an important role in these suspensions, holding the particles together, which in turn is mainly responsible for the change in the rheological properties of the suspension. These phenomena are related to capillary bridge forces and can also be observed in wet granular materials. In granular media, the addition of water results in the increase of grain cohesiveness and the lubrication of the solidsolid friction. ${ }^{12}$ The cohesion between the two granular particles that originates with the addition of water is due to the surface tension and capillary effects of the liquid.

The capillary bridges are differentiated between pendular and capillary state based on the type of fluid preferentially wetting the particles. In case of the pendular state the secondary fluid wets the particles preferentially and results in a three phase contact angle which is smaller than $90^{\circ}$, whereas in the case of the capillary state the bulk fluid preferentially wets the particles and results in a contact angle which is greater than $90^{\circ} .7$ Note that in this case, the contact angle is measured through the

Received: October 15, 2017

Revised: November 30, 2017

Published: December 14, 2017 
A
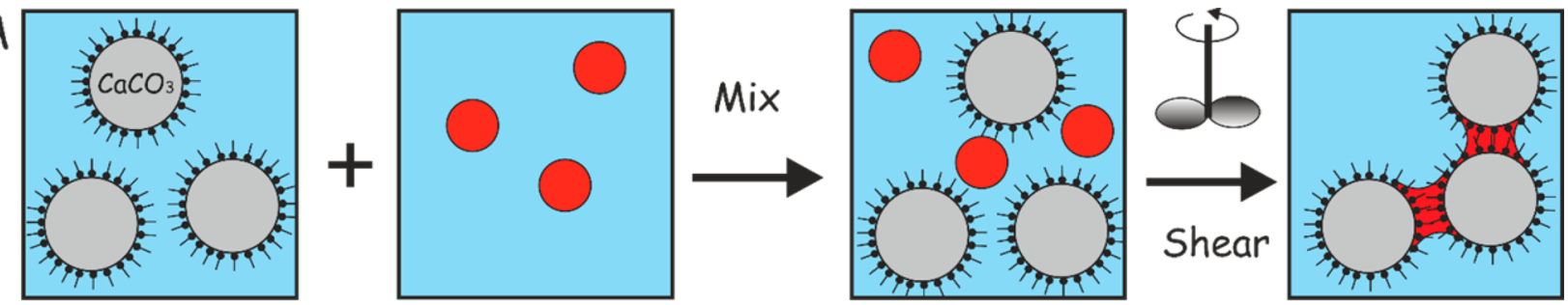

B

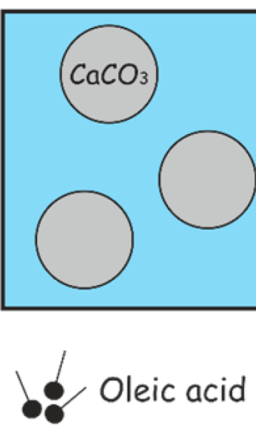

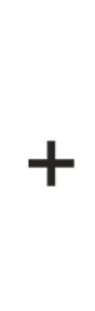
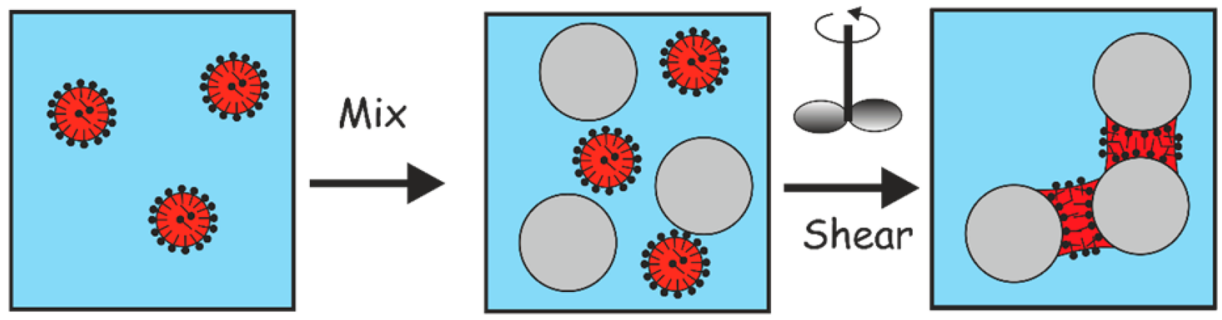

Calcium carbonate
Polar continuous phase

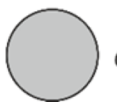

Calcium carbonate

Non-polar secondary phase

Figure 1. Schematic of the two processes used to form capillary suspensions from calcium carbonate particles dispersed in a polar liquid phase (e.g., glycerol or water). In (A) calcium carbonate particles prehydrophobized by oleic acid are dispersed in the polar continuous phase; the secondary liquid phase (oil) is added, and after applying high shear, capillary suspension is formed. In (B) bare hydrophilic calcium carbonate particles are dispersed in the polar continuous liquid phase, and then a liquid mixture of the secondary nonpolar phase with oleic acid is added. The oleic acid from the drops of the secondary liquid phase comes in contact with the calcium carbonate particles and reacts locally with their surface providing hydrophobic patches to which the secondary liquid phase adheres and forms liquid bridges and a capillary suspension. Both processes result in an increase of the suspension viscosity which can be several orders of magnitude higher than the viscosity of the same suspension without a secondary liquid phase or after prehydrophobization of the calcium carbonate particles. Note that the oil bridges and the particles are not drawn to scale, and in realty the particles would be brought in contact with each other due to the attractive capillary force. This was done in order to illustrate the surfaces where the oleic acid is accumulated during the in situ hydrophobization of particles through the oil bridges.

liquid bridge phase. The liquid bridge formed between the two particles by the secondary fluid is mainly responsible for the cohesion between the two particles. The force between these particles depends on the diameter of the particle, interfacial tension, $\gamma$, between the secondary and the continuous phase and the contact angle, $\theta$, between the solid surface in contact with the primary and the secondary fluid. ${ }^{8}$ The capillary force due to the formation a pendular capillary bridge formed between identical particles of radius $R$ in contact is given by the equation $^{7,13,14}$

$$
F_{\text {bridge }}=2 \pi R \gamma \cos \theta
$$

where $\gamma$ is the interfacial tension between the two liquid phases and $\theta$ is the contact angle of the particle contact line, measured through the polar liquid phase. Here the liquid bridge volume is assumed to be much smaller than the particle volume.

The dispersed particles can stabilize Pickering emulsions ${ }^{15}$ and can also aggregate together to form agglomerates which separate from the liquid phase. ${ }^{16}$ In the case of Pickering emulsions, the particle volume fraction is usually smaller than the droplet volume fraction. ${ }^{17}$ In capillary suspensions, the particle volume fraction is usually much larger than the volume of the individual drops of secondary fluid. ${ }^{18}$ Gelation in stable Pickering emulsions occurs due to the van der Waals attraction between solid particles stabilizing adjacent emulsion droplets. ${ }^{19}$ However, in the case of capillary suspensions, the change in rheology occurs due to the formation of capillary liquid bridges between the adjacent solid particles. The agglomeration effect of particles upon addition of secondary liquid phase of water to the oil-based suspension for sugar and solid fat particles has been previously discussed for food based applications. ${ }^{20-22}$
This results in the separation of the solids from the liquid phase resulting in faster sedimentation without the formation of capillary bridges of aqueous phase between them. There are different kinds of capillary suspensions depending on the continuous liquid phase and the secondary fluid phase. In the oil-based capillary suspension, the oil is the continuous phase and water is the secondary phase, whereas in water-based capillary suspensions it is just the opposite.

Capillary suspensions have so far been studied mainly from the perspective of food science or preparation of porous ceramic materials which utilize suspension with a nonpolar primary phase and a polar secondary (bridge forming) phase. Little work has been done on materials made with a polar primary liquid phase and less polar secondary liquid phase. Xi et al. $^{28}$ described capillary suspensions made from a large volume fraction of hollow glass spheres dispersed in water and structured by using a secondary kerosene phase. They elaborated on the control of the viscosity of these suspensions by adding very small amounts of pre-hydrophobized $\mathrm{CaCO}_{3}$ nanoparticles. Zhang et al. ${ }^{29}$ found that upon addition of the secondary immiscible fluid, the suspension viscosity is reduced through the formation of hydrophobic kerosene films. A further increase in the secondary fluid phase caused an increase in viscosity due to formation of liquid bridges and networks followed by further steep reduction in viscosity due to the formation of cells or particle-coated droplets. Capillary foams, however, also constitute "capillary structured materials" and are formed due to air entrapment in the capillary suspension during mixing. ${ }^{30,32,33}$

Recently, Das et l. $^{38}$ demonstrated thermally responsive capillary suspensions using hydrogel as a secondary (inner) 
phase bridging $\mathrm{CaCO}_{3}$ particles dispersed in oil. Hydrophilic inorganic particles such as calcium carbonate are useful structuring components in personal care products, for example, toothpastes or cosmetic creams, as are nonpolar oils such as isopropyl myristate and peppermint oil. Formation of capillary suspensions from these materials is generally a challenge as the calcium carbonate particles are too hydrophilic to form capillary bridges of the nonpolar oil in the continuous polar liquid phase. In this study, we demonstrate that capillary structured materials can be formed from hydrophilic particles suspended in a polar liquid media connected by capillary bridges formed of small amounts of an immiscible nonpolar oil. This can usually be achieved by prehydrophobization of the particles before their dispersion in the continuous polar phase (see Figure 1A). This work seeks to demonstrate that similar effect can be achieved in a single step by using non-treated solid particles and a hydrophobization agent delivered in situ from the secondary liquid phase (see Figure 1B).

Figure 1 schematically lays out the idea behind this current work, that a hydrophobizing agent can be delivered in situ from a secondary oil phase so as to favorably alter the wetting properties of the hydrophilic solid particles and facilitate the formation of capillary structured suspensions in polar continuous phase. For this purpose, oleic acid $\left(\mathrm{CH}_{3}\left(\mathrm{CH}_{2}\right)_{7} \mathrm{CH}=\mathrm{CH}\left(\mathrm{CH}_{2}\right)_{7} \mathrm{COOH}, \mathrm{OA}\right)$ was chosen as a hydrophobizing agent. The oleic acid chemically reacts with $\mathrm{Ca}^{2+}$ leaving a coat of hydrophobic oleate chains on the surface of the hydrophilic calcium carbonate particles. Though the hydrophobizing agent $(\mathrm{OA})$ can by itself form capillary bridges, in our in situ hydrophobization process it can be used as a solution in a range of water-immiscible organic liquids. Such oil-based solutions serve as the bridging liquid phase that can be incorporated into the capillary suspension in relatively small amounts. Here we study the stability and the rheological properties of the obtained capillary structured suspensions in relation to the physical properties of the system such as particle size, interfacial tension, and contact angle of the particles at the interface between the two liquid phases. We also determine the minimal concentrations of the solid particles at fixed amount of secondary (oil) phase as well as the minimal amount of secondary (oil) phase at fixed particle concentration which results in the formation of a capillary suspension in polar continuous phase.

\section{MATERIALS AND METHODS}

Materials. The particles used were ultrafine calcium carbonate (Socal 31, (mean particle diameter 0.05-0.1 $\mu \mathrm{m}$ ) and a coarser grade Socal particle range (mean particle diameter $2-3 \mu \mathrm{m}$, Solvay Specialties). The primary polar phases were Glycerol (Alfa Aeser, $99+\%)$ or deionized water obtained from an Elga MiliQ unit. The secondary nonpolar phases were isopropyl myristate (IPM, Aldrich, 98\%), Oleic acid (OA, Fisher, > 99\%), and Peppermint oil (PO, SAFC, purified by distillation). All oils were used as received.

Methods. Formation of Capillary Suspensions. The desired mass of particles were placed in a beaker with a fixed amount of the primary liquid phase. This was added to a Tefal "Fruit Sensation" blender and blended for $30 \mathrm{~s}$ on setting 1 . An aliquot of approximately $3 \mathrm{~g}$ was sampled for rheological measurement. The required amount of secondary liquid phase was added and the mixture was reblended. This was repeated until the desired number of samples with varying secondary phase concentrations at a fixed particle content had been obtained. All our rheological measurements were carried out after the samples had been desiccated by being placed in a vacuum desiccator under reduced pressure for $10 \mathrm{~min}$. We have done this because the presence of air can have a significant effect on the viscosity of the system by forming a third fluid-like phase in certain conditions. ${ }^{34}$ All samples were degassed unless otherwise stated and the concentration of the three phases are expressed as \% w/w with respect to the final system.

Rheology Measurements. Rheology was characterized using a Bohlin CVO-120 instrument fitted with a $40 \mathrm{~mm} 4^{\circ}$ cone and plate. The gap size was $150 \mu \mathrm{m}$, some 50 times the size of the largest particles and enough to ensure continuum conditions. Using a $40 \mathrm{~mm}$ parallel plates, at a fixed shear stress of $10 \mathrm{~Pa}$ a plot of applied shear stress vs the inverse of the gap over the range 100 to $500 \mu \mathrm{m}$ for $30 \%$ $\mathrm{w} / \mathrm{w}$ Socal 31 particles in water with a secondary phase of $1 \% \mathrm{w} / \mathrm{w}$ oleic acid showed the shear rate to be independent of the gap size, i.e., there was no slip in the system (see Figure S4 in the Supporting Information). Rheology measurements were done for each sample at $25{ }^{\circ} \mathrm{C}$. The sample was thermally equilibrated for $300 \mathrm{~s}$, then a controlled stress sweep from 0.1 to $1000 \mathrm{~Pa}$, in 40 steps logarithmically spaced with a delay of $40 \mathrm{~s}$ per measurement. The shear rate was measured and the calculated viscosity plotted against the applied shear. Care was taken that no material was ejected during the measurements.

Typical results for the rheology of the capillary suspension at shear stresses from 10 to $1000 \mathrm{~Pa}$ are presented as in Figure 2. Two

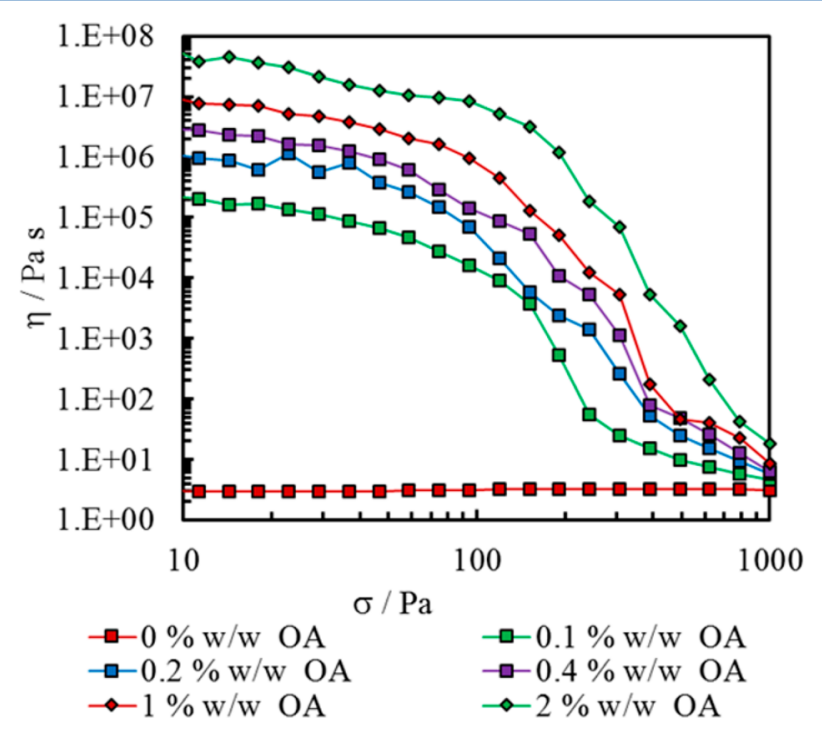

Figure 2. Viscosity $(\eta)$ vs applied shear stress $(\sigma)$ for $30 \% \mathrm{w} / \mathrm{w}$ Socal 31 dispersed in glycerol with increasing amounts of added oleic acid (OA) to the continuous phase. All concentrations are with respect to the final system. These data show that when OA is added to Socal 31 calcium carbonate particles suspended in glycerol, the rheology changes from a Newtonian fluid of relatively low viscosity (no OA) to a much more viscous, shear thinning material. Error bars are within the symbol size.

successive runs for a sample left in the rheometer between measurements revealed no significant differences, so it was deemed that a preshear process was not necessary (see Figure S5). Rheology measurements were completed within $6 \mathrm{~h}$ of the sample preparation and the samples were then left on a benchtop for at least 1 month to monitor their stability. For the complex modulus measurements, controlled frequency stress sweep oscillatory measurements were made, at $1 \mathrm{~Hz}$. The stress sweep range was altered according to the rheology of the material. The temperature was controlled to better than $\pm 0.1{ }^{\circ} \mathrm{C}$. The samples were allowed to thermally equilibrate for $10 \mathrm{~min}$ prior to measurement. No preshear was used. A fresh sample was used for each measurement.

Contact Angle Measurements. Contact angles of the calcium carbonate powder particles at the interface between the continuous and secondary liquid phases were determined by compressing the powder into a pellet using a $\mathrm{KBr}$ press. This was placed in a cuvette on a Krüss DSA-10 instrument, and stuck down with a carbon SEM 
mounting pad. The cuvette was usually filled with the less dense liquid phase, and a $20 \mu \mathrm{L}$ drop of the dense liquid phase was produced by a syringe, deposited onto the pellet surface and imaged with the Krüss DSA-10 digital camera. The DSA-10 software was used to measure the advancing contact angle through the more polar phase approximately 1 min after the drop was produced. Note that the contact angle measurement can be influenced by the surface roughness of the pellet of compressed particles. Ideally, the contact angle of the individual particles would be preferred rather than the macroscopic contact angle on the pellet surface. Such measurements can in principle be done by using the well-recognized techniques for contact angle individual particles, as the GTT ${ }^{26,35-37}$ and FreSCa. ${ }^{27}$ However, both of these techniques have their limitations. Since the Socal particles have highly irregular shape and are aggregated, we expect strong effects of hysteresis in the contact angle of the individual particles due to pinning of the three-phase contact line on the edges of the $\mathrm{CaCO}_{3}$ microcrystals. As the Socal particle aggregates are also nonspherical, the application of both techniques for individual particles is challenging and we do not expect to produce more accurate results than those the pellet-based method. The same approach has also been adopted by other authors working on capillary suspensions. ${ }^{28}$

Equilibrium Interfacial Tension Measurements. Equilibrium interfacial tensions between the continuous and the secondary liquid phases were determined using a Krüss K11 tensiometer with Du Nuoy ring, with temperature set at $25^{\circ} \mathrm{C}$. The quoted tensions are determined from the equation describing the linear portion of a plot of interfacial tension, $\gamma$, versus the inverse square root of time, where the intercept is taken to be the equilibrium interfacial tension. Measurements were usually taken over 15-20 min. All glassware was cleaned by immersion in $1 \mathrm{M}$ potassium hydroxide solution in ethanol for $24 \mathrm{~h}$ followed by thorough rinsing with MiliQ water. The ring was burned in a blue Bunsen flame until glowing red prior to each use.

Pretreatment of Calcium Carbonate Particles with Oleic Acid. The desired amount of OA was dissolved in $100 \mathrm{~mL}$ of ethanol in a beaker. A sample of $40 \mathrm{~g}$ of the calcium carbonate powder (Socal 31 or coarser grade Socal) was added and stirred vigorously. The beaker was then placed open in a water bath at $70{ }^{\circ} \mathrm{C}$ overnight to evaporate off the ethanol. The calcium carbonate after this treatment appeared to be a free-flowing white powder, indistinguishable by eye from the untreated powder. This treatment allowed the calcium carbonate particles to be prehydrophobized by the OA treatment.

SEM Imaging of Flash-Freezed and Freeze-Dried Capillary Suspensions. The SEM images were obtained using a Zeiss Evo 60 Scanning Electron Microscope. The samples were mounted on a specific holder and plunged frozen into slushy nitrogen. Slushing reduces the $\mathrm{N}_{2}$ temp by about $14{ }^{\circ} \mathrm{C}$ to $-210{ }^{\circ} \mathrm{C}$ and minimizes boiling upon sample freezing. The samples were then sublimed at -70 ${ }^{\circ} \mathrm{C}$ and $10^{-6} \mathrm{mbar}$ for $7 \mathrm{~min}$. They were transferred onto a cryo-SEM cool stage in the preparation chamber under vacuum. The sample was then fractured and coated with an electro-conductive coating by using an integrated Sputter Coater. Finally, they were transferred into the SEM chamber all under vacuum. The vacuum was regulated with a leak valve connected to an argon supply. The operating pressure was 2-4 Pa. A direct current voltage (about $2 \mathrm{kV}$ ) was applied to the source material $(\mathrm{Au} / \mathrm{Pd}$ target) which ionizes the argon gas. Argon ions were accelerated toward the target, ejecting metal atoms in the process. These metal atoms coat the samples which were rotated at about $0.5 \mathrm{rev} / \mathrm{s}$ during sputtering. The sputtering process was terminated automatically by the thickness monitor at a coating thickness of approximately $2-3 \mathrm{~nm}$.

\section{RESULTS AND DISCUSSION}

Formation of a Capillary Suspension in Polar Continuous Phase. Socal 31 (calcium carbonate) is a hydrophilic material, readily dispersed into polar liquids such as water or glycerol. Indeed, the affinity of these liquids for the calcium carbonate is such that some hydrophobization of the particle surface is required for the secondary liquid phase, a less polar oil, to attach to the particle surface and form strong capillary bridges that would lead to capillary structured suspension. Pure oleic acid (OA) was initially chosen as the secondary oil phase as carboxylic acids are expected to chemically react with the calcium carbonate surface, grafting it with hydrophobic oleate groups, thus giving hydrophobic anchoring patches for the secondary liquid phase to form capillary bridges. Figure 2 shows the viscosity vs the applied shear stress curves for $30 \% \mathrm{w} / \mathrm{w}$ Socal 31 particles dispersed in glycerol, with small amounts of OA added. It is also evident from Figure 2 that without the addition of OA, the Socal 31 particle suspension in glycerol behaves as a Newtonian fluid at the same particle concentration. Upon addition of $0.1 \% \mathrm{w} / \mathrm{w}$ $\mathrm{OA}$ to the suspension, the material is clearly gel-like, exhibiting a high viscosity at low stress and a yield stress. The viscosity at low stress increases with the increase of the percentage of $\mathrm{OA}$ added to this system. This can be explained as the excess of OA behaves as a secondary liquid phase in this capillary structured suspension which can form liquid capillary bridges between the Socal 31 particles. Note that OA is insoluble in glycerol but can chemically react with the Socal 31 surface to form a layer of oleate. The particles surface in contact with the OA droplets are in situ hydrophobized which allows them to attach to the particles and provides anchoring patches for the capillary bridges. The number of capillary bridges is expected to increase with the increase of the amount of OA in the system. As the viscosity of this capillary structured system depends on the number of capillary bridges between their particle network and the value of the maximum capillary force per capillary bridge, $F_{\text {bridge }}$ (see eq 1 ), one may envisage that within certain range of OA concentrations, the increase in the amount of the secondary liquid phase would lead to an increase in the number of bridges as well as the viscosity of the suspension. We did the measurement for 30\% w/w Socal 31 suspension in water as a continuous phase instead of glycerol which demonstrate very similar share thinning behavior-the results are presented in Figure S1.

Figure 3 summarizes the data for the viscosity of the Socal 31 suspension in glycerol at fixed shear stress as a function of the $\mathrm{OA}$ concentration. Underneath are images of the materials produced, demonstrating that without OA there is no capillary structuring while where minute amounts of $\mathrm{OA}$ has been added, the material is gel-like and can be sculpted. The equilibrium interfacial tension at $25{ }^{\circ} \mathrm{C}$ between glycerol and the $\mathrm{OA}$ is $7.9 \pm 0.1 \mathrm{mN} \mathrm{m}^{-1}$. The advancing and receding contact angles of glycerol on a tablet of Socal 31 immersed in $\mathrm{OA}$, measured through the glycerol, are $118 \pm 2^{\circ}$ and $110 \pm 2^{\circ}$, respectively.

In this system, the contact angle of the primary phase with the particle is greater than $90^{\circ}$, so a pendular state bridge structure has been formed. Replacing glycerol with water in this system gives very similar results (Figure 4). Figure 5 shows the freeze-fracture scanning electron microscope images of freezedried suspensions of the Socal 31 particles in water. In the case where no $\mathrm{OA}$ is present in the system, the particles appear as a randomly aggregated in the dispersion (Figure 5a). In the presence of OA, there is an order and 3D structuring of the Socal 31 particles. In this case, the system appears to form a bicontinuous network of particles suspended in water (Figure $5 \mathrm{~b})$. The higher magnification SEM image shows that the particles are packed much closer together and more compact in the system with OA present than when simply dispersed in water without secondary liquid phase added. We do not expect that the structure is changing during imaging as the suspension 

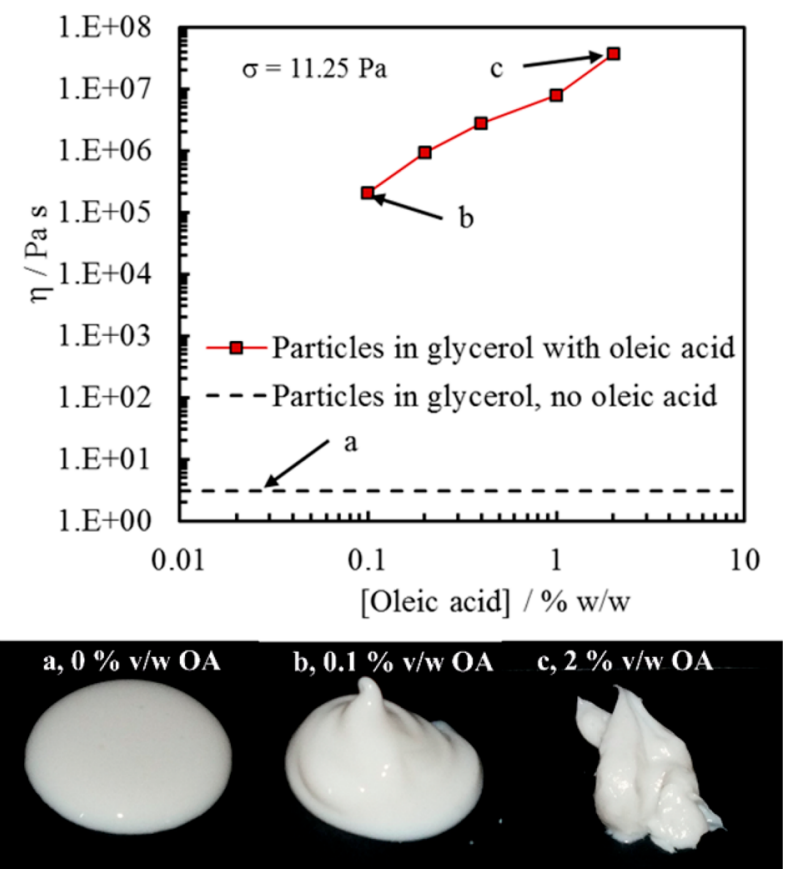

Figure 3. Glycerol based suspension viscosity $(\eta)$ at shear stress $\sigma=$ $11.25 \mathrm{~Pa}$ vs concentration of OA. The system consists of $30 \% \mathrm{w} / \mathrm{w}$ Socal 31 particles dispersed in glycerol. The dotted line is particles with no oleic acid. The enclosed digital images labeled (a), (b), and (c) show the suspensions corresponding to $0,0.1$, and $2 \% \mathrm{w} / \mathrm{w}$. The increase in viscosity as a function of the OA concentration results in a marked change in visual appearance of the suspension from a flowable liquid (a, no OA) to a gel which can be sculpted (c, $2 \%$ w/w OA).

samples have been flash-frozen in liquid nitrogen and then freeze-dried. Domenech and Velankar ${ }^{31}$ have also reported similar-looking structures in capillary suspensions where the particles form capillary aggregates network and some hierarchical structures within it. However, their systems are not made by in situ hydrophobized particles through the secondary liquid phase as in our work.

It has been previously found that calcium carbonate particles coated in $\mathrm{OA}$ and dispersed in water will form aggregates, driven by the attraction between the hydrophobic particle surfaces in water. ${ }^{23}$ Such aggregation could in principle cause the increase in viscosity observed with the addition of OA to Socal 31 powder dispersed in a polar liquid. However, here we show that this is not the case by pretreating the Socal 31 powder with just enough $\mathrm{OA}$ to form a monolayer on the particle surface and dispersing them into glycerol. We compared the rheology of this suspension with the case when the same amount of OA was added to a suspension of untreated Socal 31 calcium carbonate in glycerol (Figure 6). We estimated that $0.04 \mathrm{~mL}$ of OA per gram of Socal 31 particles is required to form a densely packed monolayer of $\mathrm{OA}$ completely coating the particle surfaces (see the SEI for the enclosed calculation of the amount of OA needed to fully hydrophobize the Socal 31 particles). This amount is rather an overestimate as a densely packed monolayer of OA cannot be formed under such conditions. In practice, it was found that $0.0015 \mathrm{~mL}$ OA per gram of Socal 31 particle was sufficient (the equivalent of $0.03 \% \mathrm{w} / \mathrm{w}$ in a system with $20 \% \mathrm{w} / \mathrm{w}$ particles). Figure 6 shows that for $0.03 \% \mathrm{w} / \mathrm{w}$ OA and $20 \% \mathrm{w} / \mathrm{w}$ Socal 31 calcium carbonate, the pretreated particles dispersed in glycerol
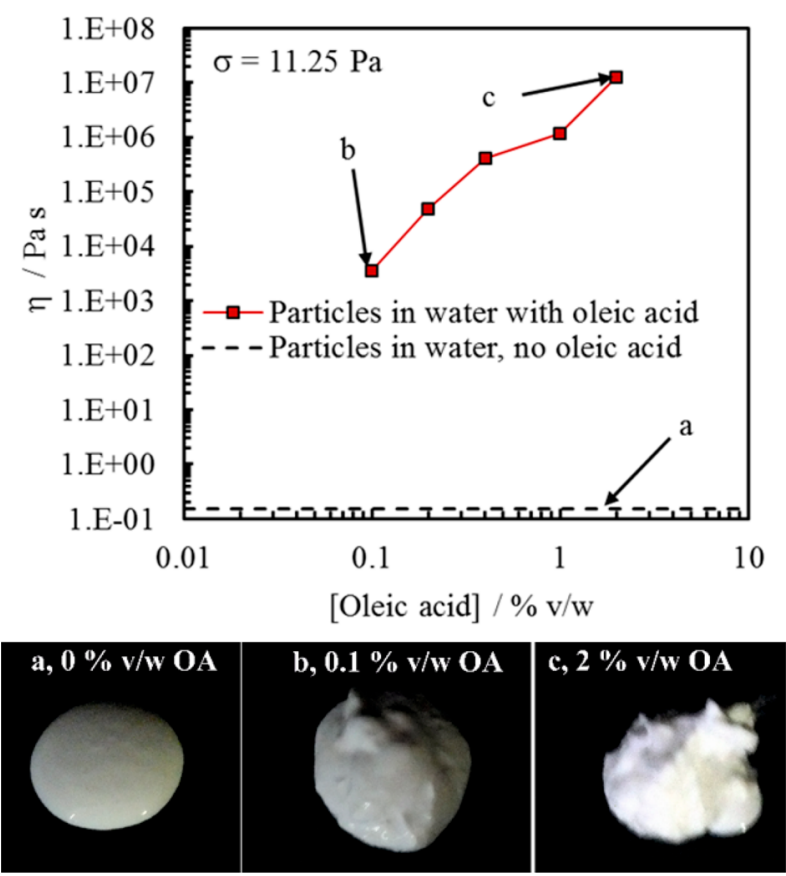

Figure 4. Aqueous suspension viscosity $(\eta)$ at shear stress $(\sigma)=11.25$ Pa vs concentration of OA. The system consists of $30 \% \mathrm{w} / \mathrm{w}$ Socal 31 particles dispersed in water with different concentrations of added OA. The dashed line represents the particles suspension with no oleic acid. Points (a), (b), and (c) show $0,0.1$, and $2 \% \mathrm{w} / \mathrm{w}$ OA corresponding to the three images of the suspension underneath. Note the steep increase in the suspension's viscosity as a function of the OA concentration, and the marked change in its visual appearance from a flowable liquid (a, no OA) to a gel which can be sculpted (c, $2 \% \mathrm{w} / \mathrm{w}$ $\mathrm{OA})$.

behaves as a relatively low viscosity Newtonian fluid over the shear stress range studied.

Thus, it was concluded that there is no significant change in the rheology of the particles suspended in glycerol due to the presence of the preadsorbed oleic acid. The same amount of OA added to a dispersion of untreated Socal 31 particles forms a shear thinning material. The advancing contact angle of a glycerol drop on a tablet of OA-treated Socal 31 particles under IPM was $78 \pm 2^{\circ}$, compared with $15 \pm 2^{\circ}$ with untreated Socal 31 particles measured on a compressed tablet of this material. This suggests that OA has been adsorbed onto the particle surfaces and prehydrophobized the calcium carbonate particles. The advancing contact angle of a drop of OA with untreated and pretreated Socal 31 immersed in glycerol (measured through the more polar phase, i.e., the glycerol) were $118 \pm 2^{\circ}$ and $135 \pm 2^{\circ}$, respectively. This nonzero contact angle shows that the excess of $\mathrm{OA}$ in the drop does not spread over the pretreated Socal 31 particles to form a bilayer or a film. Therefore, any amount of OA in excess of that required to form an oleate monolayer would be present in the form of droplets able to form liquid OA capillary bridges between the Socal 31 particles.

For the system where OA was added to the dispersion of untreated particles in glycerol, the $\mathrm{OA}$ is unlikely to coat the entire surface of the particles as they are already in contact with glycerol. Thus, even a small amount of OA is expected to be present in the suspension as drops, able to form capillary bridges between patches of particle surface in situ hydrophobized by OA oil drops when they come in contact with the 


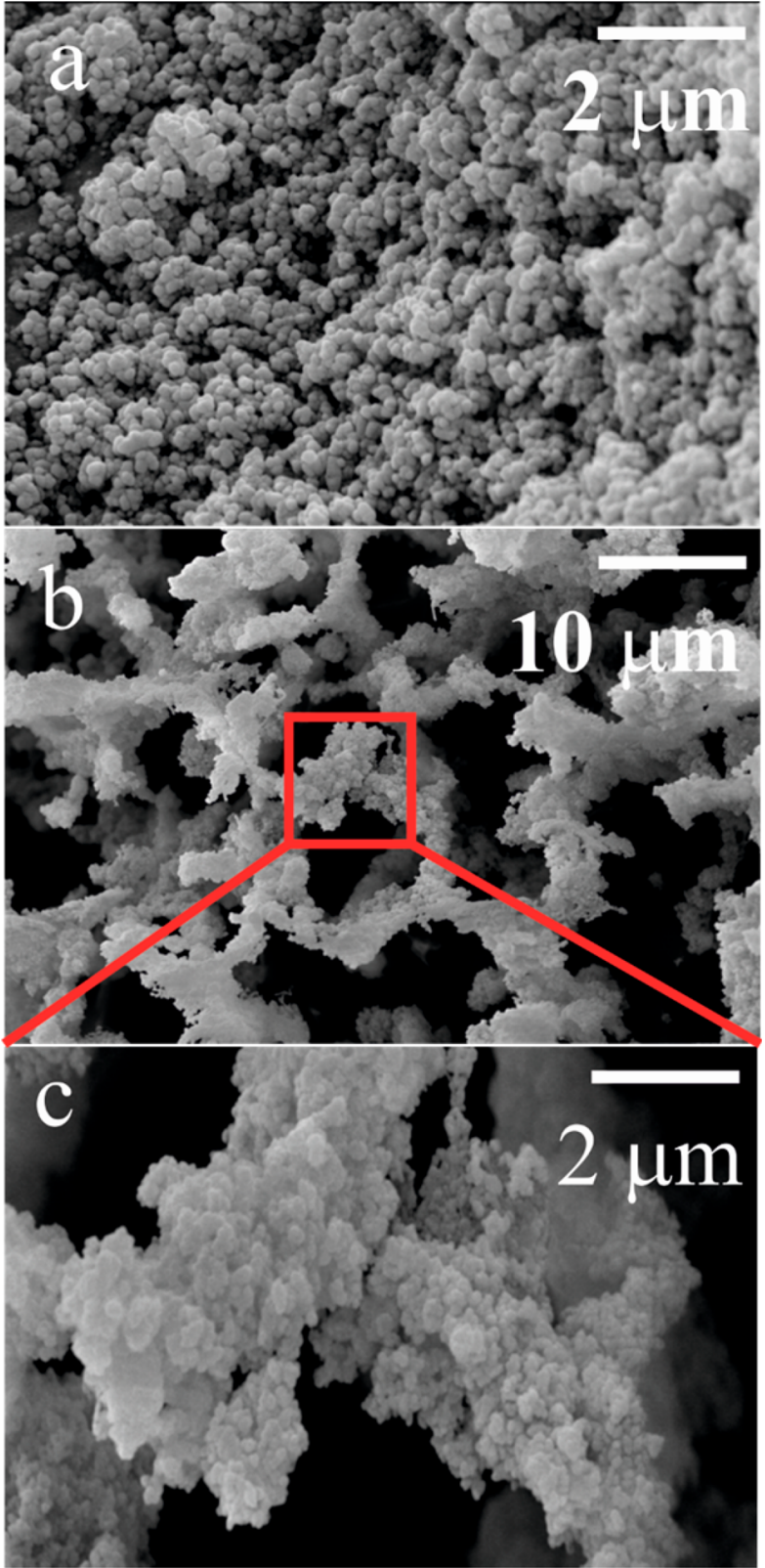

Figure 5. Scanning electron microscopy image of $20 \%$ w/w Socal 31 which has originally been dispersed in water (a) with no added secondary liquid phase and (b) with $2 \% \mathrm{w} / \mathrm{w} \mathrm{OA}$ as a secondary phase. The samples were freeze-fractured then freeze-dried prior to imaging, so the voids in the image are where the aqueous phase has been in the original sample. Note that in absence of secondary liquid phase the Socal 31 calcium carbonate particles produce randomly aggregated dispersion in the aqueous phase. However, the particle suspension becomes capillary structured after the addition of the $\mathrm{OA}$ as a secondary liquid phase, with what appears to be a bicontinuous system of particle/OA network suspended in the aqueous phase. The magnified image of the highlighted region (c) shows that within the particle network forming the porous structure, the individual particles are packed more closely together than in the absence of the oleic acid (a). At this magnification the capillary bridges of free OA are too small to be seen on the SEM image.

particles during the mixing process. Figure 6 clearly shows that the rheology of a dispersion of untreated Socal 31 calcium carbonate in glycerol is strongly altered by the subsequent addition of a very small amount of $\mathrm{OA}$, while pretreating the

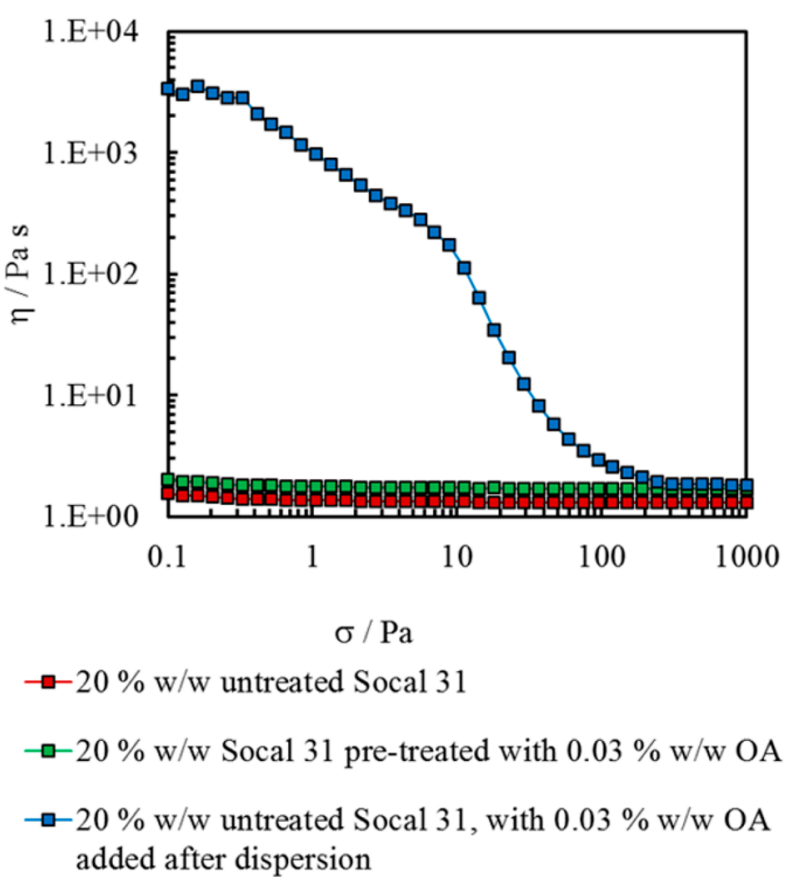

Figure 6. Viscosity $(\eta)$ vs applied shear stress $(\sigma)$ for a suspension of $20 \% \mathrm{w} / \mathrm{w}$ Socal 31 dispersed in glycerol. The study includes for comparison the cases of suspension without oleic acid (OA) and with $0.03 \% \mathrm{w} / \mathrm{w} \mathrm{OA}$ added either as a pretreatment to the powder particles or directly to the already formed dispersion of Socal 21 particles in glycerol. All concentrations are presented with respect to the final system. Note that where OA is present on the particle surface as a hydrophobizing monolayer with no excess of $\mathrm{OA}$ available to form capillary bridges, the rheology of the system resembles the case of a dispersion without OA, i.e., there is no marked increase in the suspension viscosity due to hydrophobic interactions between the prehydrophobized Socal 31 particles.

Socal 31 with the same amount of OA before mixing with the glycerol phase does not give any substantial increase in the suspension viscosity as no capillary bridges are formed between the particles.

In Situ Particle Hydrophobization by Oleic Acid Delivered through an Inert Secondary Oil Phase. Isopropyl myristate (IPM) and peppermint oil (PO, a mixture of menthol, menthone, methyl acetate, eucalyptol, limonene, $p$-pinene, and $p$-caryophyllene $)^{24}$ are not expected to chemically interact with the surface of hydrophilic Socal 31 particles when dispersed in the continuous polar phase, and should therefore be unable to form capillary structured materials. Figure 7 shows that for a Socal 31 suspension in glycerol, the addition of up to $2 \% \mathrm{w} / \mathrm{w}$ of IPM results in an almost Newtonian fluid over the shear stress range shown, without significant difference to rheology of the Socal 31 suspension in glycerol with no added IPM. Using peppermint oil (PO) instead of IPM gave a very similar result (see Figure S2). The variation in the data is a measure of the experimental error.

Figure 8 shows that for both oils there is no significant increase in the viscosity at a shear stress of $11.25 \mathrm{~Pa}$ of a suspension of $30 \%$ Socal 31 particles in glycerol with the addition of either oil. If an oil bridge is formed between two particles approaching the oil drop in water (or glycerol), the dynamic contact angle on the particle surface would correspond to the receding contact angle measured through the more polar phase (water, glycerol). However, in reality, during the capillary 


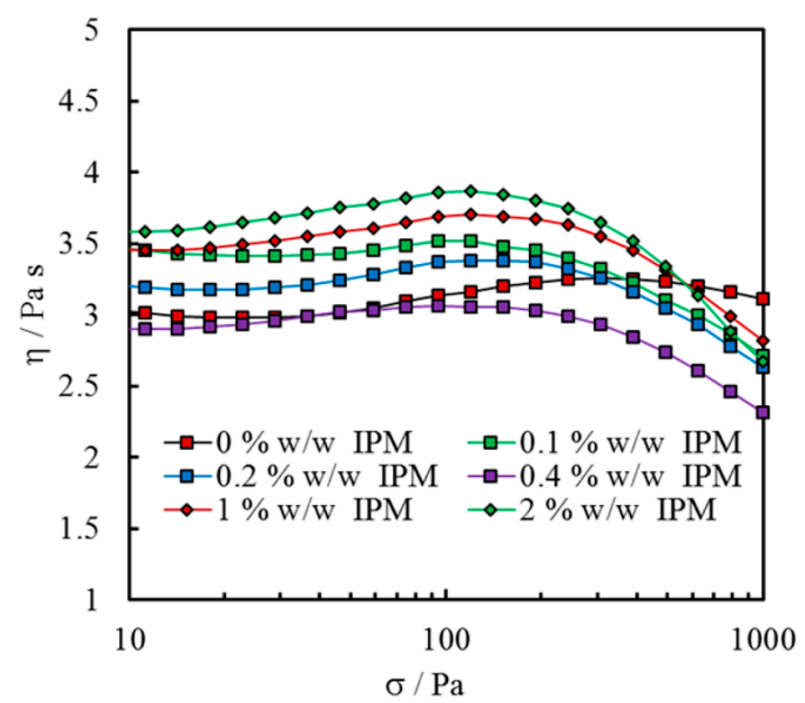

Figure 7. Viscosity $(\eta)$ vs applied shear stress $(\sigma)$ for $30 \% \mathrm{w} / \mathrm{w}$ Socal 31 dispersed in glycerol. The graph shows the effect of the varying the concentration of added secondary phase, isopropyl myristate (IPM) on the viscosity. All concentrations are with quoted with respect to the final system. The system contains hydrophilic solid particles of Socal 31 and does not contain a hydrophobizing agent. These results indicate that the nonadhering IPM liquid drops do not form capillary bridges between the hydrophilic calcium carbonate particles (Socal 31) which results in a low viscosity even at significant percentage of IPM.

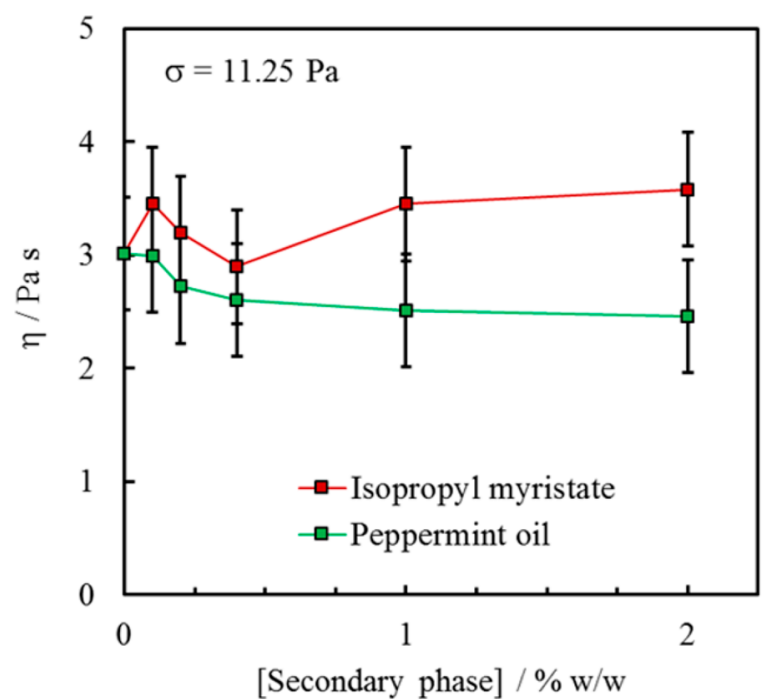

Figure 8. Viscosity $\eta$ at $\sigma=11.25 \mathrm{~Pa}$ vs the concentration of the secondary phase for $30 \%$ Socal 31 particles dispersed in glycerol. The graph shows the effect of adding isopropyl myristate (IPM) and peppermint oil (PO) as a secondary phase and indicates that the nonadhering IPM and PO do not form capillary bridges between the calcium carbonate particles. All concentrations are with respect to the final system.

suspension preparation, the applied shear causes the oil bridges to break and reform again multiple times during the shearing. Thus, the maximal force an oil bridge could withstand is more likely related to the advancing contact angle (measured through the polar phase) as the polar phase is advancing in such dynamic situation. Therefore, we compare systematically only the advancing contact angles on Socal 31 particle pellets at different $\mathrm{OA}$ concentration in the oil phase. The results show that the increase of the OA concentration in the oil leads to a substantial increase of the contact angle as the Socal 31 surface in contact with the oil becomes more hydrophobized. Figure 9

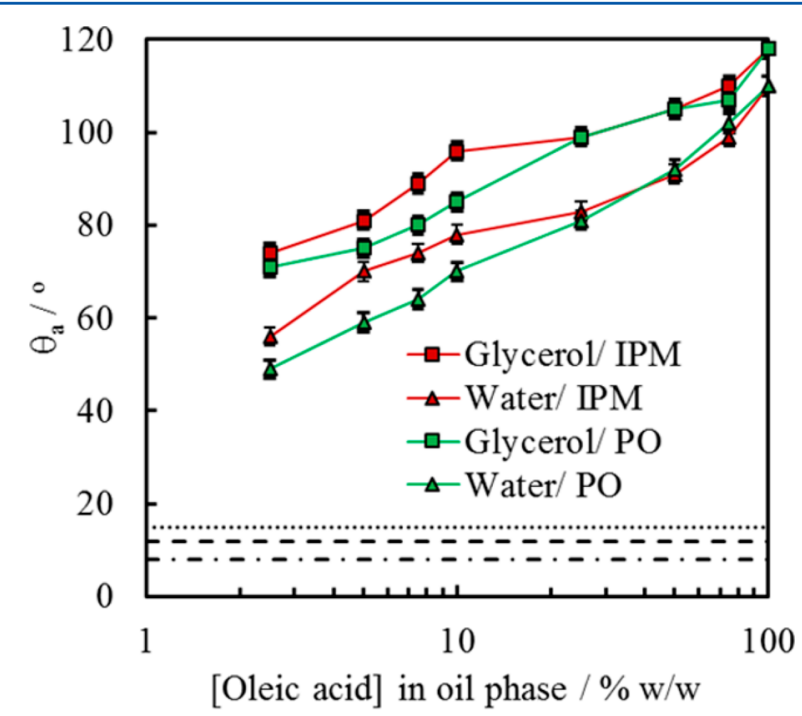

Figure 9. Advancing contact angle $\left(\theta_{a}\right)$ of a sessile drop of polar phase (water or glycerol) on a tablet of compressed Socal 31 particles immersed in the oil phase vs the concentration of oleic acid in the oil phase for four combinations of polar and nonpolar (oil) phases: glycerol/isopropyl myristate (IPM), water/IPM, glycerol/peppermint oil (PO), and water/PO. The dashed lines show the advancing contact angle for the system without oleic acid present for glycerol/IPM $\left(15^{\circ}\right.$, dotted line $)$, water/IPM $\left(12^{\circ}\right.$, dashed line $)$, glycerol/PO $\left(12^{\circ}\right.$, dashed line), and water/PO $\left(8^{\circ}\right.$, dash-dot line $)$. These results demonstrate that $\mathrm{OA}$ delivered through the sessile oil drop hydrophobizes the surface of the Socal 31 particles in contact with the oil.

shows the advancing contact angles of both glycerol and water drops on a pellet of compressed Socal 31 powder immersed in the oil phase with different concentrations of OA. Here the OA has been added to either IPM or PO. The surface of the particles has a greater affinity for polar fluids than for IPM or PO. The Socal 31 particle contact angle increases significantly with increasing the concentration of OA in the oil.

This is ascribed to the lowering of the interfacial tension between the polar liquid and the oil by adsorption of OA. This effect is combined with contact line pinning on the hydrophobized patch of solid surface due to adsorption of OA on the Socal 31 particle surface. At low OA concentration, the contact angle of the particles is less than $90^{\circ}$ (measured through the polar liquid phase), indicating a capillary state bridges might be formed, as the contact angle of the particles increases above $90^{\circ}$ it might be inferred that a pendular types of liquid bridges are created at high OA concentrations. Figure 10 shows the rheology of $30 \% \mathrm{w} / \mathrm{w}$ Socal 31 suspended in glycerol with $1 \%$ $\mathrm{w} / \mathrm{w}$ oil phase, where the proportion of OA in the oil phase has been varied. Shear thinning material was formed at as little as $2.5 \% \mathrm{OA}$ in the IPM oil phase $(0.025 \% \mathrm{w} / \mathrm{w}$ OA with respect to the final formulation). The viscosity of this system at low shear stress is several orders of magnitude higher than the viscosity of the same particles in glycerol with either zero oil or with $\mathrm{PO}$ alone without added OA. A similar result was obtained for systems with PO (see Figure S3).

The OA concentration has got a huge effect on the rheology of the system. Note that the IPA/ $\mathrm{CaCO}_{3} /$ glycerol system does not form effective capillary suspension as the nonhydrophobized Socal 31 particles are too hydrophilic, i.e., their contact 


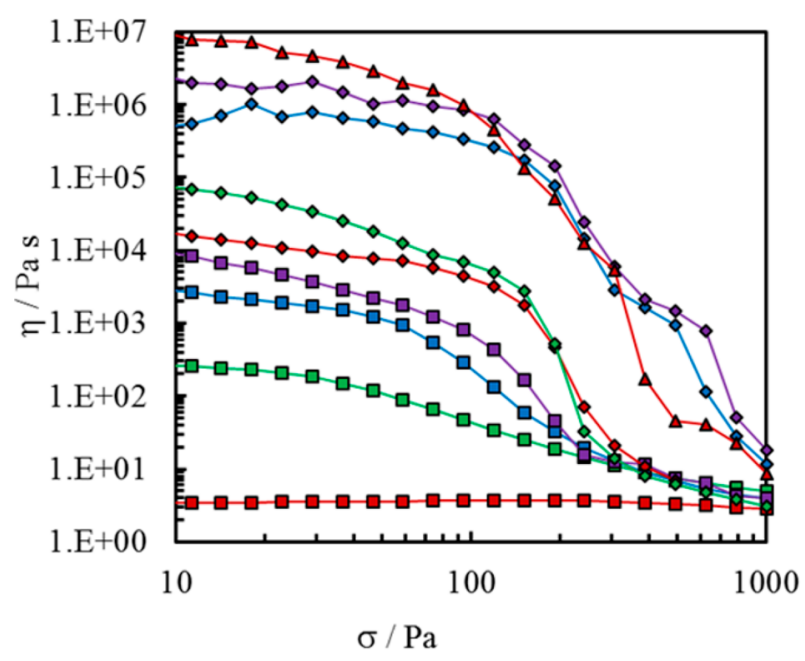

$\rightarrow-0 \% \mathrm{v} / \mathrm{w}$ OA, $1 \% \mathrm{w} / \mathrm{w}$ IPM

$\rightarrow-0.025 \% \mathrm{w} / \mathrm{w}$ OA, $0.975 \% \mathrm{w} / \mathrm{w}$ IPM

$\rightarrow-0.05 \% \mathrm{w} / \mathrm{w}$ OA, $0.95 \% \mathrm{w} / \mathrm{w}$ IPM

$\rightarrow-0.075 \% \mathrm{w} / \mathrm{w}$ OA, $0.925 \% \mathrm{w} / \mathrm{w}$ IPM

$\rightarrow-0.1 \% \mathrm{w} / \mathrm{w}$ OA, $0.9 \% \mathrm{w} / \mathrm{w}$ IPM

$\rightarrow-0.25 \% \mathrm{w} / \mathrm{w}$ OA, $0.75 \% \mathrm{w} / \mathrm{w}$ IPM

$\rightarrow 0.5 \% \mathrm{w} / \mathrm{w}$ OA, $0.5 \% \mathrm{w} / \mathrm{w}$ IPM

$\rightarrow 0.75 \% \mathrm{w} / \mathrm{w}$ OA, $0.25 \% \mathrm{w} / \mathrm{w}$ IPM

$\triangle-1 \% \mathrm{w} / \mathrm{w}$ OA, $0 \% \mathrm{w} / \mathrm{w}$ IPM

Figure 10. Viscosity $(\eta)$ vs the applied shear stress $(\sigma)$ for $30 \% \mathrm{w} / \mathrm{w}$ Socal 31 dispersed in glycerol with $1 \% \mathrm{w} / \mathrm{w}$ secondary liquid phase. The results are presented for a series of secondary liquid phases of isopropyl myristate (IPM) with increasing concentration of OA. All concentrations are quoted with respect to the final system. The graph shows that for a fixed secondary liquid phase concentration, the rheology changes very sharply with the increase of OA concentration in the secondary phase, ranging from Newtonian fluid $(0 \% \mathrm{w} / \mathrm{w}$ OA, $1 \% \mathrm{ww} / \mathrm{w}$ IPM) to a viscous shear thinning material $(1 \% \mathrm{w} / \mathrm{w}$ OA, $0 \%$ ww/w IPM).

angle (measured through the glycerol phase) is only about $15^{\circ}$. However, as one can see from Figure 9, above $10 \%$ OA in the oil phase, the particle contact angle increases over $90^{\circ}$, i.e., the oil bridges become pendular.

The more OA is contained in the oil bridges, the higher the viscosity of the Socal 31 suspension. At $100 \%$ OA as oil, the viscosity at low shear stress is more than $10^{6}$ times higher than the suspension containing the same particle concentration and the pure oil (IPM) at the same volume fraction (see Figure 10).

Figure 11a presents the viscosity at a shear stress of $11.25 \mathrm{~Pa}$ for both IPM and PO as a secondary phase. The viscosity of the PO-doped systems is generally greater than the IPM-doped systems. The sample viscosity would depend on the capillary bridge force, $F_{\text {bridge }}$, and the number of capillary bridges formed. According to eq $1, F_{\text {bridge }}$ increases with interfacial tension and with the cosine of the contact angle. IPM-doped systems generally have a higher interfacial tension with the polar phase (see Table 2) and a slightly lower contact angle with the solid than the PO-doped systems (Figure 9), so it is expected that $F_{\text {bridge }}$ be significantly lower for the PO-doped systems than for the IPM-doped systems. A reasonable physical explanation for the effect could be that there are more capillary bridges formed in the PO-doped systems than in the IPM-doped systems as the former has much lower $\mathrm{o} / \mathrm{w}$ interfacial tension which would facilitate the breaking of the oil phase to smaller drops at the same shear (see Table 2).
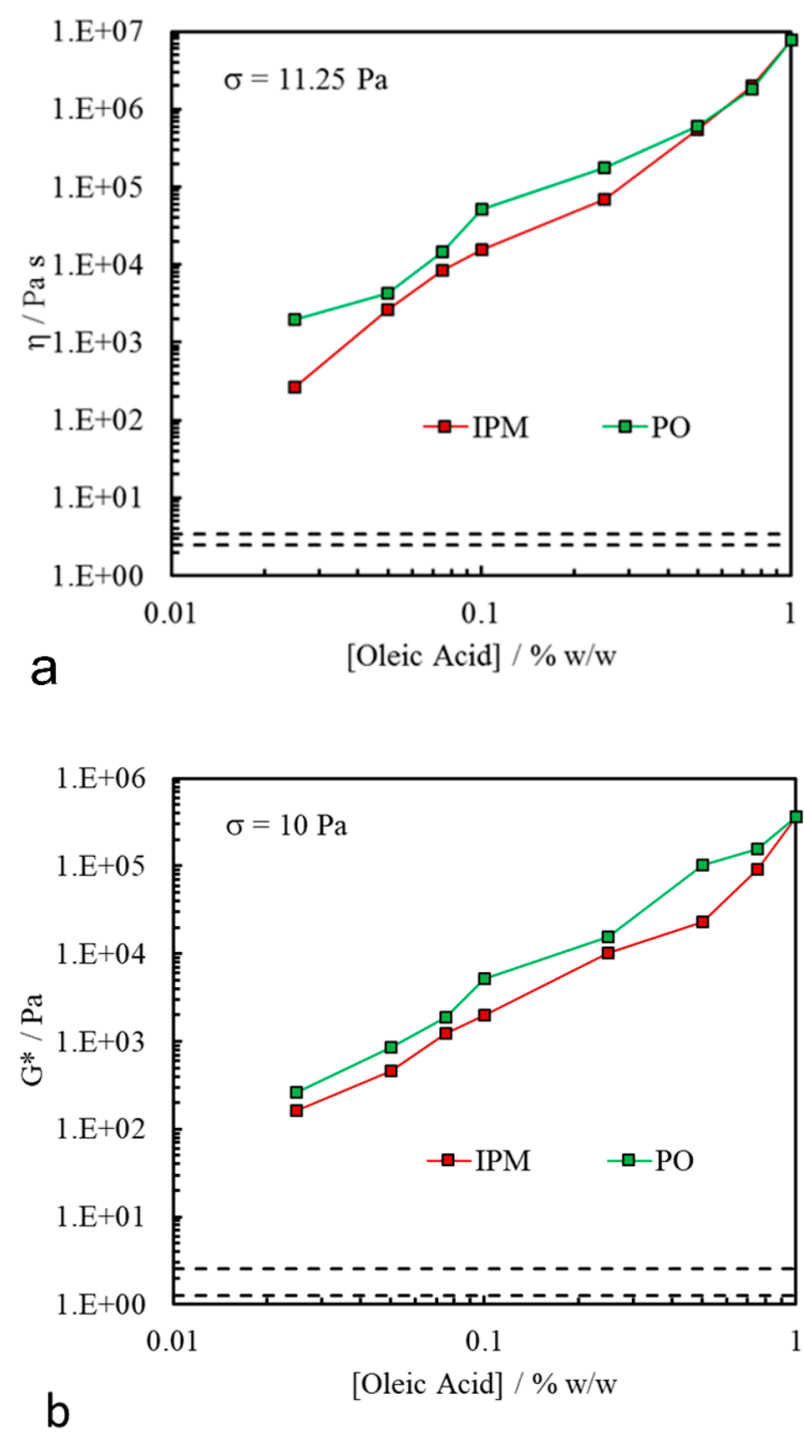

Figure 11. (a) Viscosity $(\eta)$ at shear stress $(\sigma)=11.25 \mathrm{~Pa}$ vs the concentration of oleic acid for a system of $30 \%$ Socal 31 particles dispersed in glycerol with $1 \% \mathrm{w} / \mathrm{w}$ secondary (oil) phase (IPM or $\mathrm{PO})$. So for $0.5 \% \mathrm{w} / \mathrm{w}$ oleic acid, $0.5 \% \mathrm{w} / \mathrm{w}$ of the nonadsorbing inert oil is also present in the system. The dashed lines represent the viscosity for a system with $0 \% \mathrm{w} / \mathrm{w}$ oleic acid for IPM $(\eta=3.5 \mathrm{~Pa} \mathrm{~s})$ and PO $(\eta=2.5 \mathrm{~Pa} \mathrm{~s})$. (b) Complex modulus $\left(\mathrm{G}^{*}\right)$ at shear stress $(\sigma)$ $=10 \mathrm{~Pa}$ vs the concentration of oleic acid for a system of $30 \%$ Socal 31 particles dispersed in glycerol with $1 \% \mathrm{w} / \mathrm{w}$ secondary (oil) phase (IPM or PO). The dashed lines represent the viscosity for a system with $0 \% \mathrm{w} / \mathrm{w}$ oleic acid for $\mathrm{IPM}\left(\mathrm{G}^{*}=2.6 \mathrm{~Pa}\right)$ and $\mathrm{PO}\left(\mathrm{G}^{*}=1.3 \mathrm{~Pa}\right)$. All concentrations are given with respect to the final system.

The viscosity of the samples increased with the increase of OA concentration and hence increased with the increase of the advancing contact angle of the Socal 31 particles in this system. This shows that a hydrophobizing agent has successfully been delivered from the oil phase to the particle surfaces, rendering them hydrophobic enough that oil drops can adhere to the particles and form stable oil bridges.

Figure 11b shows the effect of the OA concentration on the complex modulus for two different oils (IPM and PO), where the capillary suspension of Socal 31 in glycerol contains $1 \% \mathrm{w} /$ $\mathrm{w}$ oil phase in total. The concentration of the OA within the oil phase is varied similarly to Figure 11a. The RHS data points on Figure $11 \mathrm{a}, \mathrm{b}$ corresponds to $1 \% \mathrm{w} / \mathrm{w}$ pure OA in glycerol. The 
results show that both the low shear viscosity and complex modulus of the systems increase with the increase of OA concentration in the secondary oil phase in the case of both PO and IPM.

Effect of the Primary Liquid Phase, the Particle Size, and the Oil Phase on the Rheology and Stability of Capillary Suspensions. The formation of a capillary suspension requires all three phases (primary and secondary liquid phase, solid particles) to be present in a specific ratio between them. Firstly, if not enough secondary liquid phase is present to form liquid capillary bridges and networks of bridged particles, settling of the calcium carbonate particles can be observed. Second, if there is an excess of the secondary oil phase, then the particles begin to form Pickering emulsion drops which results in creaming and the formation of a top layer rich in particles and oil drops. These instabilities appear within a few hours of the three components being subjected to high shear as illustrated in Figure 12. It is only when the ratio of

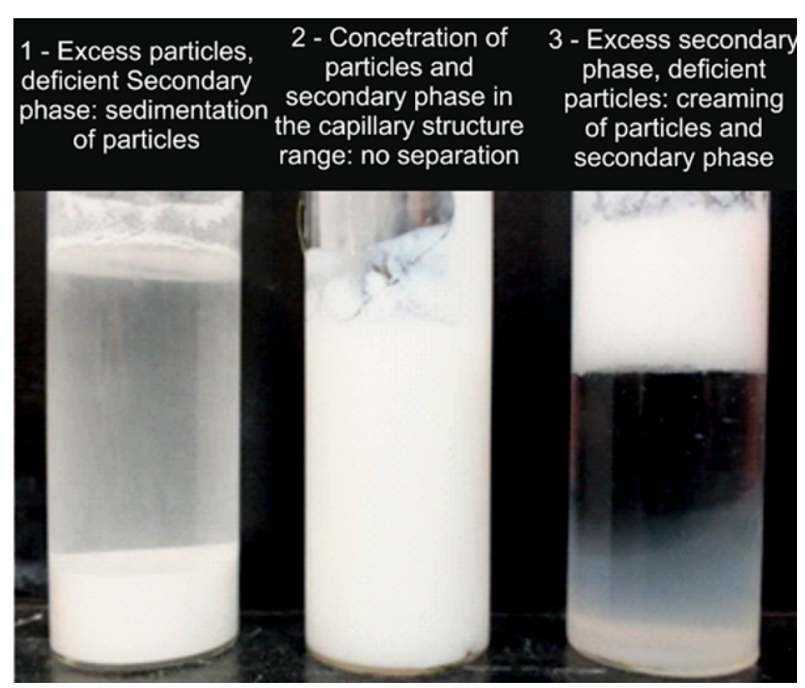

Figure 12. Digital photographs showing the stability of aqueous suspensions of $30 \% \mathrm{w} / \mathrm{w}$ Socal 31 calcium carbonate, with different amounts of $0.1 \% \mathrm{w} / \mathrm{w}$ oleic acid in $0.9 \% \mathrm{w} / \mathrm{w}$ IPM added as a secondary liquid phase. The images illustrate the three types of system observed: (1) sedimentation of the solid particles in a system with not enough secondary liquid phase to form a capillary structured material; (2) no visible signs of phase separation, i.e., a stable capillary suspension; (3) creaming of the particles and the oil phase in a system with an excess of secondary (oil) phase and due to the formation of an o/w Pickering emulsion.

the three components is within narrow boundaries that capillary structured materials are formed, which appear to show no signs of phase separation or visible instability over a period of months. This was checked for all the systems reported here. We determined the minimum secondary liquid phase (oil) concentration required to form a stable capillary structured material for a fixed concentration of particles dispersed in the polar liquid phase. In addition, we determined the minimum particle concentration for a fixed concentration of secondary liquid phase required to form a stable capillary suspension. These measurements were carried out for two different grades of calcium carbonate particles, Socal 31 and a coarser grade Socal, both manufactured by precipitation methods. The primary difference between the two grades of
Socal is the particle size. Socal 31 has a mean particle diameter of $0.05-0.1 \mu \mathrm{m}$, the coarser grade Socal was $2-3 \mu \mathrm{m} .^{25}$

Table 1 summarizes the lower capillary structuring limits of the particle and oil concentration for each of the eight systems and also shows the viscosity of the capillary suspensions at fixed shear stress. From this data, it is clear that as the particle average size increases, so the minimal particle concentration required to form capillary suspension also increases. For water as a primary phase, the minimum particle concentration required to form capillary suspension increases from 10 to $20 \% \mathrm{w} / \mathrm{w}$; for glycerol it increases from $5 \% \mathrm{w} / \mathrm{w}$ to $10 \% \mathrm{w} / \mathrm{w}$. This is explained by consideration of the particle surface area available for the formation of liquid bridges in each system. Smaller particles would have a much larger surface area than the equivalent mass of larger particles, which corresponds to much greater possible number of liquid bridges formed between the small particles per unit volume of the suspension. One would expect capillary structuring to take place at a lower particle concentration for the smaller particles.

For suspensions formed from an equivalent amount of larger particles, the concentration of the secondary liquid phase required to form capillary suspension increases. With the increase of the average particle diameter, larger oil bridges are formed and higher concentration of the secondary oil phase is required to prevent particle sedimentation under gravity and phase separation of the suspension. One sees that the viscosity of the capillary suspension decreases with the increase of the average particle diameter. The number of liquid bridges per unit volume present in a capillary suspension of smaller particles would be much greater than that for larger particles. Since the capillary suspension viscosity is proportional to the product of the capillary bridge force and the number of bridges, it is expected that a system with smaller particles would have a greater viscosity than the same system with larger particles at the same weight percentage of particles. The viscosity of the glycerol based capillary structured systems is generally greater than this of water based systems. This is due to the viscosity of the primary phase, some $70 \% \mathrm{w} / \mathrm{w}$ of the materials, being over 1000 times greater for glycerol than water. The viscosity of IPM systems is generally greater than that for PO systems. Table 2 shows the variation of the interfacial tension of IPM or PO with water or glycerol as a function of OA content.

Since the capillary bridge forces between the solid particles are controlled by the value of the primary liquid/secondary liquid interfacial tension, $F_{\text {bridge }}$ would be greater for IPM systems than for $\mathrm{PO}$, and the viscosity of a system with a similar number of capillary bridges will be greater for IPM systems than that for PO systems.

\section{CONCLUSIONS}

We have successfully prepared capillary suspensions of hydrophilic calcium carbonate microparticles using a polar primary liquid phase and a very low concentration of an immiscible oil as a secondary phase after application of high shear. Two different polar liquid phases were used, namely, glycerol and water. We used IPM and PO doped with OA as a secondary fluid phase. Our method was based on local in situ hydrophobization of the calcium carbonate particles by delivering $\mathrm{OA}$ as a hydrophobizing agent directly through the oil phase. This method gives capillary suspensions whose viscosity is several orders of magnitude higher than the original calcium carbonate suspension in the polar phase without the addition of OA-doped secondary oil phase or with the non- 
Table 1. Summary of Lowest Particle and Oil Phase Concentrations Required to Form Capillary Suspensions, and the Viscous Response of the Material, for Primary Phases of Water and Glycerol, Two Calcium Carbonate Particles of Varying Size and Two Oil Phase

\begin{tabular}{|c|c|c|c|c|c|}
\hline $\begin{array}{l}\text { Primary } \\
\text { phase }\end{array}$ & Type of particles & $\begin{array}{l}\text { Secondary } \\
\text { liquid phase } \\
\text { composition }\end{array}$ & $\begin{array}{c}\text { Lowest concentration of } \\
\text { secondary phase at } 30 \% \mathrm{w} / \mathrm{w} \\
\text { particles } / \% \mathrm{w} / \mathrm{w}\end{array}$ & $\begin{array}{l}\text { Lowest particles } \\
\text { concentration at } 0.4 \% \mathrm{w} / \mathrm{w} \\
\text { secondary phase } / \% \mathrm{w} / \mathrm{w}\end{array}$ & $\begin{array}{c}\text { Viscosity at } 11.25 \mathrm{~Pa} \text { shear stress, } \\
30 \% \mathrm{w} / \mathrm{w} \text { particles, } 0.4 \% \mathrm{w} / \mathrm{v} \\
\text { secondary phase }\end{array}$ \\
\hline Water & Socal $31(0.05-0.1 \mu \mathrm{m})$ & $\begin{array}{l}10 \% \text { oleic acid/ } \\
90 \% \text { IPM }\end{array}$ & 0.2 & 10 & 48300 \\
\hline Water & Socal $31(0.05-0.1 \mu \mathrm{m})$ & $\begin{array}{l}10 \% \text { oleic acid/ } \\
90 \% \text { Pepper- } \\
\text { mint oil }\end{array}$ & 0.2 & 10 & 10235 \\
\hline Water & Coarser grade Socal range $(2-3 \mu \mathrm{m})$ & $\begin{array}{l}10 \% \text { oleic acid/ } \\
90 \% \text { IPM }\end{array}$ & 0.4 & 20 & 5205 \\
\hline Water & Coarser grade Socal range $(2-3 \mu \mathrm{m})$ & $\begin{array}{l}10 \% \text { oleic acid/ } \\
90 \% \text { Pepper- } \\
\text { mint oil }\end{array}$ & 0.2 & 20 & 1598 \\
\hline Glycerol & Socal $31(0.05-0.1 \mu \mathrm{m})$ & $\begin{array}{l}10 \% \text { oleic acid/ } \\
90 \% \text { IPM }\end{array}$ & 0.2 & 5 & 298900 \\
\hline Glycerol & Socal $31(0.05-0.1 \mu \mathrm{m})$ & $\begin{array}{l}10 \% \text { oleic acid/ } \\
90 \% \text { Pepper- } \\
\text { mint oil }\end{array}$ & 0.2 & 5 & 174700 \\
\hline Glycerol & Coarser grade Socal range $(2-3 \mu \mathrm{m})$ & $\begin{array}{l}10 \% \text { oleic acid/ } \\
90 \% \text { IPM }\end{array}$ & 0.2 & 10 & 21840 \\
\hline Glycerol & Coarser grade Socal range $(2-3 \mu \mathrm{m})$ & $\begin{array}{l}10 \% \text { oleic acid/ } \\
90 \% \text { Pepper- } \\
\text { mint oil }\end{array}$ & 0.2 & 10 & 7256 \\
\hline
\end{tabular}

Table 2. Equilibrium Interfacial Tension $(\gamma)$ of IPM or Peppermint oil with Water or Glycerol As a Function of the Oleic Acid Concentration

\begin{tabular}{ccccc} 
& \multicolumn{4}{c}{ Interfacial tension $(\gamma) / \mathrm{mN} \mathrm{m}^{-1}$} \\
\cline { 2 - 5 } $\begin{array}{c}\text { Oleic acid concentration } \\
\text { in oil phase/\% w/w }\end{array}$ & $\begin{array}{c}\text { IPM/ } \\
\text { water }\end{array}$ & $\begin{array}{c}\text { Peppermint } \\
\text { oil/water }\end{array}$ & $\begin{array}{c}\text { IPM/ } \\
\text { Glycerol }\end{array}$ & $\begin{array}{c}\text { Peppermint } \\
\text { oil/Glycerol }\end{array}$ \\
0 & 22.3 & 6.1 & 20.3 & 3.9 \\
2.5 & 20.6 & 7.9 & 17.3 & 4.2 \\
10 & 15.7 & 8.1 & 15.1 & 4.7 \\
100 & 8.9 & 8.9 & 7.9 & 7.9 \\
\hline
\end{tabular}

doped secondary phase alone. We also performed series of experiments to prove that the sharp increase in the viscosity is not due to hydrophobization of the calcium carbonate particles alone. The characteristic high viscosity arises from the presence of oil bridges and not from the hydrophobic attraction between the particles once coated with OA.

We show that increasing the calcium carbonate particle size decreases the number of oil bridges present, leading to a lower viscosity than a system with smaller particles and a higher number of oil bridges. Glycerol has a higher initial viscosity than water, so glycerol based systems have a greater viscosity than water based systems. We also studied the effect of the interfacial tension between the polar liquid phase and the oil on the viscosity of the capillary suspension. We found that IPMbased secondary phase gave higher viscosity of the capillary structured materials compared with PO-based secondary phase at identical conditions. This is attributed to the much lower interfacial tension at the $\mathrm{PO} /$ water and $\mathrm{PO} /$ glycerol interface which corresponds to lower values of the capillary bridge forces between the particles. We also investigated the role of the contact angle on the calcium carbonate particle surface in the capillary suspension. The latter varies from $60^{\circ}$ to $120^{\circ}$ (measured through the polar liquid) depending on the concentration of the $\mathrm{OA}$ in the secondary liquid phase which correspond to change of the oil bridges from capillary to pendular state.
We explored the minimal concentrations of secondary liquid phase required for capillary suspension formation at fixed percentage of particles in the primary liquid phase for two different sizes of particles (Socal 31 and coarser grade Socal range) dispersed in glycerol or water. Capillary structured suspensions were obtained at secondary phase concentration exceeding $0.2 \% \mathrm{w} / \mathrm{v}$ at $30 \% \mathrm{w} / \mathrm{w}$ Socal 31 while it was $0.4 \% \mathrm{w} /$ $\mathrm{v}$ at $30 \% \mathrm{w} / \mathrm{w}$ coarser grade Socal particles which indicates that larger particles need higher concentration of secondary liquid phase to form enough oil bridges to match the viscosity of a capillary suspension of the finer particles at the same conditions. Similarly, at fixed concentration of the secondary liquid phase, the minimal particle concentration was determined to be $10 \% \mathrm{w} / \mathrm{w}$ for Socal 31 and $20 \% \mathrm{w} / \mathrm{w}$ for coarser grade Socal particles at $0.4 \% \mathrm{w} / \mathrm{w}$ secondary liquid phase. Again these results show that finer particles gave capillary suspensions at lower particle concentrations. Capillary suspensions in polar liquid phases are very versatile systems where the rheology of the system and its properties can be controlled by very small amount of added secondary liquid phase and can find applications in a range of food products as well as home and personal care formulations.

\section{ASSOCIATED CONTENT}

\section{Supporting Information}

The Supporting Information is available free of charge on the ACS Publications website at DOI: 10.1021/acs.langmuir.7b03589.

Calculation of volume oleic acid per gram of Socal 31; complete data sets of viscosity $(\eta)$ vs applied shear stress $(\sigma)(\mathrm{PDF})$

\section{AUTHOR INFORMATION}

\section{Corresponding Author}

*E-mail: V.N.Paunov@hull.ac.uk. Phone +44 1482 465660/ Fax: +44 1482466410.

ORCID 8

Timothy S. Dunstan: 0000-0001-5628-4629 
Anupam A. K. Das: 0000-0003-1948-8811

Simeon D. Stoyanov: 0000-0002-0610-3110

Vesselin N. Paunov: 0000-0001-6878-1681

\section{Author Contributions}

The manuscript was written through contributions of all authors. All authors have given approval to the final version of the manuscript.

\section{Notes}

The authors declare no competing financial interest.

\section{ACKNOWLEDGMENTS}

T.D., A.D., and V.P. acknowledge funding of this work from Unilever R\&D Vlaardingen, The Netherlands. The authors appreciated the technical help from Mr Tony Sinclair at the University of Hull Microscopy Suite with the SEM sample preparation and imaging.

\section{REFERENCES}

(1) Koos, E.; Willenbacher, N. Capillary forces in suspension rheology. Science 2011, 331, 897-900.

(2) Koos, E.; Kannowade, W.; Willenbacher, N. Restructuring and aging in a capillary suspension. Rheol. Acta 2014, 53, 947-957.

(3) Van Kao, S.; Nielsen, L. E.; Hill, C. T. Rheology of concentrated suspensions of spheres. II. Suspensions agglomerated by an immiscible second liquid. J. Colloid Interface Sci. 1975, 53, 367-373.

(4) Gögelein, C.; Brinkmann, M.; Schröter, M.; Herminghaus, S. Controlling the formation of capillary bridges in binary liquid mixtures. Langmuir 2010, 26, 17184-17189.

(5) Koos, E.; Johannsmeier, J.; Schwebler, L.; Willenbacher, N. Tuning suspension rheology using capillary forces. Soft Matter 2012, 8, 6620-6628.

(6) Koos, E.; Kannowade, W.; Willenbacher, N. Restructuring and aging in a capillary suspension. Rheol. Acta 2014, 53, 947-957.

(7) Hoffmann, S.; Koos, E.; Willenbacher, N. Using capillary bridges to tune stability and flow behavior of food suspensions. Food Hydrocolloids 2014, 40, 44-52.

(8) Dittmann, J.; Koos, J.; Willenbacher, N. Ceramic Capillary Suspensions: Novel Processing Route for Macroporous Ceramic Materials. J. Am. Ceram. Soc. 2012, 96, 391-397.

(9) Allain, C.; Cloitre, M.; Wafra, M. Aggregation and sedimentation in colloidal suspensions. Phys. Rev. Lett. 1995, 74, 1478-1481.

(10) Buscall, R; McGowan, I. J.; Mills, P. D. A.; Stewart, R. F.; Sutton, D.; White, L. R.; Yates, G. E. The rheology od strongly floculated suspensions. J. Non-Newtonian Fluid Mech. 1987, 24, 183202.

(11) Dijkstra, M. Computer simulations of charge and steric stabilised colloidal suspensions. Curr. Opin. Colloid Interface Sci. 2001, 6, 372-382.

(12) Mitarai, N.; Nori, F. Wet granular materials. Adv. Phys. 2006, 55, $1-45$.

(13) Rabinovich, Y. I.; Esayanur, M. S.; Moudgil, B. M. Capillary forces between two spheres with a fixed volume liquid bridge: Theory and experiment. Langmuir 2005, 21, 10992-10997.

(14) Schubert, H. Capillary forces - modeling and application in particulate technology. Powder Technol. 1984, 37, 105-116.

(15) Aveyard, R.; Binks, B. P.; Clint, J. H. Emulsions stabilised solely by colloidal particles. Adv. Colloid Interface Sci. 2003, 100-102, 503546.

(16) Leonard, W. G.; Greer, R. T.; Markuszewski, R.; Wheelock, T. D. Coal desulfurization and deashing by oil agglomeration. Sep. Sci. Technol. 1981, 16, 1589-1609.

(17) Binks, B. P. Particles as surfactants-similarities and differences. Curr. Opin. Colloid Interface Sci. 2002, 7, 21-41.

(18) Koos, E.; Willenbacher, N. Particle configurations and gelation in capillary suspensions. Soft Matter 2012, 8, 3988-3994.
(19) Akartuna, I.; Studart, A. R.; Tervoort, E.; Gonzenbach, U. T.; Gauckler, L. J. Stabilization of Oil-in-Water Emulsions by Colloidal Particles Modified with Short Amphiphiles. Langmuir 2008, 24, 71617168.

(20) Johansson, D.; Bergenståhl, B. The influence of food emulsifiers on fat and sugar dispersions in oils. I. Adsorption, sedimentation. J. Am. Oil Chem. Soc. 1992, 69, 705-717.

(21) Johansson, D.; Bergenståhl, B. The influence of food emulsifiers on fat and sugar dispersions in oils. II. Rheology, colloidal forces. J. Am. Oil Chem. Soc. 1992, 69, 718-727.

(22) Johansson, D.; Bergenståhl, B. The influence of food emulsifiers on fat and sugar dispersions in oils. III. Water content, purity of oils. J. Am. Oil Chem. Soc. 1992, 69, 728-733.

(23) Kawashima, Y.; Handa, T.; Takeuchi, Y.; Tanaka, H. Spherical agglomeration of calcium carbonate dispersed in aqueous medium containing sodium oleate. Powder Technol. 1986, 46, 61-66.

(24) Schmidt, E.; Bail, S.; Buchbauer, G.; Stoilova, I.; Atanasova, T.; Krastanov, A.; Jirovetz, L. Chemical composition, olfactory evaluation and antioxidant effects of essential oil from Mentha x piperita. Nat. Prod. Commun. 2009, 8, 1107-1112.

(25) Manufacturer's (Solvay B.V.) MSDS data sheet.

(26) Paunov, V. N. Novel method for determining the three-phase contact angle of colloid particles adsorbed at air-water and oil-water interfaces. Langmuir 2003, 19, 7970-7976.

(27) Isa, L.; Lucas, F.; Wepf, R.; Reimhult, E. Measuring singlenanoparticle wetting properties by freeze-fracture shadow-casting cryoscanning electron microscopy. Nat. Commun. 2011, 2, 438.

(28) Xu, M.; Liu, H.; Zhao, H.; Li, W. How to Decrease the Viscosity of Suspension with the Second Fluid and Nanoparticles? Sci. Rep. 2015, 3, 3137.

(29) Zhang, J.; Zhao, H.; Li, W.; Xu, M.; Liu, H. Multiple Effects of the Second Fluid on Suspension Viscosity. Sci. Rep. 2015, 5, 16058.

(30) Zhang, Y.; Wang, S.; Zhou, J.; Benz, G.; Tcheimou, S.; Zhao, R.; Behrens, S. H.; Carson-Meredith, J. Capillary Foams: Formation Stages and Effects of System Parameters. Ind. Eng. Chem. Res. 2017, 56, 9533-9540.

(31) Domenech, T.; Velankar, S. S. On the rheology of pendular gels and morphological developments in paste-like ternary systems based on capillary attraction. Soft Matter 2015, 11, 1500-1516.

(32) Velankar, S. S. A non-equilibrium state diagram for liquid/fluid/ particle mixtures. Soft Matter 2015, 11, 8393-8403.

(33) Zhang, Y.; Wu, J.; Wang, H.; Carson-Meredith, J.; Behrens, S. H. Stabilization of Liquid Foams through the Synergistic Action of Particles and an Immiscible Liquid. Angew. Chem., Int. Ed. 2014, 53, 13385-13389.

(34) Zhang, Y.; Allen, M. C.; Zhao, R.; Deheyn, D. D.; Behrens, S. H.; Carson-Meredith, J. Langmuir 2015, 31, 2669-2676.

(35) Cayre, O. J.; Paunov, V. N. Three-Phase Contact Angles of Colloid Gold and Silica Particles at Air-Water and Decane-Water Surfaces Studied with the Gel Trapping Technique“. Langmuir 2004, 20, 9594-9599.

(36) Arnaudov, L. A.; Cayre, O. J.; Stoyanov, S. D.; Cohen-Stuart, M.; Paunov, V. N. Nanoimprinting Method for Characterization of the Wettability of Individual Nanoparticles Adsorbed at Liquid Surfaces. Phys. Chem. Chem. Phys. 2010, 12, 328-331.

(37) Sharp, E. L.; Al-Shehri, H.; Horozov, T. S.; Stoyanov, S. D.; Paunov, V. N. Adsorption of shape-anisotropic and porous particles at the air-water and the decane-water interface studied by the gel trapping technique. RSC Adv. 2014, 4, 2205-2213.

(38) Das, A. A. K.; Dunstan, T. S.; Starck, P.; Stoyanov, S. D.; Paunov, V. N. Thermally Responsive Capillary Suspensions. ACS Appl. Mater. Interfaces 2017, No. 10.1021/acsami.7b11358, DOI: 10.1021/ acsami. $7 \mathrm{~b} 11358$. 